\title{
Targeting Thioredoxin System with an Organosulfur Compound, Diallyl Trisulfide (DATS), Attenuates Progression and Metastasis of Triple-Negative Breast Cancer (TNBC)
}

\author{
Yuping Lium ${ }^{a, b, c}$ Yang Zhao ${ }^{a, c} \quad$ Zhonghong Weia,c Li Tao ${ }^{a, c}$ Xiaobo Shenga,c \\ Siliang Wang ${ }^{a, c}$ Jiao Chen ${ }^{b, d}$ Junshan Ruan ${ }^{a, c}$ Zhaoguo Liu ${ }^{a, c} \quad$ Yuzhu Cao ${ }^{a, c}$ \\ Yunlong Shan ${ }^{a, c}$ Aiyun Wang ${ }^{a, c}$ Wenxing Chen ${ }^{a, c} \quad$ Yin Lu $^{a, c}$ \\ ajiangsu Key Laboratory for Pharmacology and Safety Evaluation of Chinese Materia Medica, School \\ of Pharmacy, Nanjing University of Chinese Medicine, Nanjing, ${ }^{b}$ Affiliated Hospital of Integrated \\ Traditional Chinese and Western Medicine, Nanjing University of Chinese Medicine, Nanjing, cliangsu \\ Collaborative Innovation Center of Traditional Chinese Medicine (TCM) Prevention and Treatment of \\ Tumor, Nanjing University of Chinese Medicine, Nanjing, 'Laboratory of Cellular and Molecular Biology, \\ Jiangsu Province Academy of Traditional Chinese Medicine, Nanjing, China
}

\section{Key Words}

DATS • Metastatic breast cancer • Oxidative stress • Thioredoxin system • Trx-1

\begin{abstract}
Background/Aims: Metastasis is the leading cause resulting in high mortality in triple negative breast cancer (TNBC) patients. Cancer cells are skilled at utilizing thioredoxin (Trx) system as an efficient antioxidant system to counteract oxidative damage, facilitating the occurrence of metastasis. Here, we identified an organosulfur compound named DATS isolated from garlic, that inhibits the expression of Trx-1 and the enzyme activity of Trx reductase in breast cancer cells. Methods: Tissue microarray of breast cancer patients and immunohistochemical method were used to analyze the role of Trx- 1 in breast cancer metastasis. Spotaneous metastasis model and experimental metastasis model combined with HE staining, immunohistochemistry were used to verify in vivo anti-metastatic effect of DATS as well as its regulation on thioredoxin. Western blot, immunofluorescence, redox state assessment and detection of enzyme activity were employed to determine the effect of DATS on thioredoxin system. Trx-1 siRNA interference was used to investigate the conclusive evidence that Trx-1 was the target of DATS. Results: In agreement with reduced Trx-1 nuclear translocation from cytoplasm by DATS, the production of reduced form of Trx-1 was dramatically decreased. Furthermore, in vivo,
\end{abstract}

Yuping Liu and Yang Zhao contributed equally to this work. 
DATS administration was observed to significantly suppress spontaneous and experimental metastasis in nude mice. Delivery of DATS also resulted in decreased expression of Trx-1 as the direct target, as well as expression of NF-KB and MMP2/9 in primary tumor and lung tissue. Notably, the effects of DATS on the expression of downstream metastasis-associated genes were mediated by Trx-1, as demonstrated by the combination use of DATS and Trx-1 siRNA. Conclusion: Collectively, this present study indicates that targeting Trx system with DATS may provide a promising strategy for treating metastasis of TNBC.

\section{Introduction \\ (C) 2018 The Author(s) \\ Published by S. Karger AG, Basel}

Garlic (Allium) has been employed as a flavouring and medicinal agent for hundreds of years. Epidemiological studies have indicated an inverse correlation between garlic consumption and the progression of cancer [1]. Organic sulphur compounds (OSCs), the major ingredients derived from garlic, mainly include diallyl sulfide (DAS), diallyl disulfide (DADS), diallyl trisulfide (DATS), all of which exert biological effects by providing sulfur radical and sulfhydryl group. DATS possesses the most sulphur atoms and has been well demonstrated to be the most powerful component among the OSCs due to its three-sulfur functional group [2]. It has been well known that DATS exhibits a variety of biological effects, such as anti-oxidant, anti-tuberculosis, and anti-inflammation [3]. Interestingly, recent studies have shown that DATS is able to inhibit tumor cell cycle progression and induce apoptosis, as well as reverse drug resistance and inhibit angiogenesis by downregulating VEGF [4]. The purpose of this study was to gain insight into the role of DATS in breast cancer progression.

Breast cancer is the most frequently diagnosed malignant disease and the leading cause of cancer death resulting from local recurrence and distant metastasis among women [57]. Human triple-negative breast cancer (TNBC), characterized by tumors deficient in the expression of estrogen receptor (ER), progesterone receptor (PR) and human epidermal growth factor receptor 2 (HER-2) [8], is recognized as a major clinical challenge owing to its molecular and genetic heterogeneity as well as the lack of well-defined specific therapeutic targets $[9,10]$. Current treatment approaches for TNBC include platinum agents, taxanes, anthracycline, novel microtubule stabilizing agents and other DNA-damaging regimens [11]. Nevertheless, these strategies are likely to be limited by intrinsic or acquired resistance and produced cross-resistance to other therapeutic agents [12]. Therefore, continued research aim at identifying novel potential therapeutic routes for improving outcomes of women with TNBC appear to be extremely valuable.

Growing evidence has suggested that redox status of tumor cells that counteracts excessive reactive oxygen species (ROS) is required for the spread of cancer [13]. Tumor cells are inclined to protect themselves from oxidative damage via maintaining their redox status to survive and successfully metastasize to distant organs [14]. Activation of redox signaling pathways facilitates breast cancer progression and is closely linked to poor prognosis in breast cancer patients [15]. Thioredoxin (Trx) system, mainly consisted of Trx, thioredoxin reductase (TrxR) and nicotinamide adenine dinucleotide phosphate (NAPDH), plays a pivotal role in modulating cellular thiol/disulfide redox status $[16,17]$. Thioredoxin-1 (Trx-1) as the predominant isoform of Trx is a $12 \mathrm{kD}$ ubiquitous protein containing a redox-active disulfide/ dithiol and its cysteine residues are converted to the oxidized intramolecular disulfide bond state (Trx-1-S2) in a reaction [18]. However, the oxidized state can be rapidly restored to reduced state (Trx-1-(SH)2) that is critical to control the protein function with the assistance of TrxR [19]. The reduced state of Trx-1 is of particular importance to regulate a series of transcription factors (e.g. NF- $\mathrm{KB}$ and AP1) through translocation into the nucleus from cytoplasm, promoting the expression of downstream target genes [20]. Notably, expression and activity of Trx are also tightly controlled by the endogenous inhibitor thioredoxininteracting protein (TXNIP), which binds to Trx and restrict its capability to interact with other proteins [21].

It has been recently documented that Trx-1 that secreted by tumor cells is responsible for aggressive tumor progression and reduced patient survival. Overexpression of Trx1 in tumor cells results in the inhibition of apoptosis signal-regulating kinase 1 (ASK1), modulating JNK/p38 signaling pathway and suppressing tumor cell apoptosis $[22,23]$. 
Further, Trx- 1 promotes the expression of hypoxia-inducible factor $1 \alpha$ (HIF- $1 \alpha$ ), leading to an increase in vascular endothelial growth factor (VEGF) production and augmented tumor angiogenesis [24, 25]. More importantly, Trx-1 overexpression stimulates the expression of matrix metalloproteinase MMP-2 and MMP-9 through NF- $\kappa B$, AP-1 and SP-1 elements, disrupts the MMP/TIMP equilibrium and mediates MMP involvement in a more invasive phenotype [26].

By the results of our previous work it was demonstrated that DATS plays a critical role in preventing tumor cell migration and invasion in vitro, at least in part, via regulating the expression of MMP2/9 [2]. However, the underlying mechanism of the inhibition by DATS of tumor progress requires further elucidation. In the present study, we provided experimental evidence to reveal that DATS-induced suppression effect on breast cancer metastasis is mediated by targeting Trx system, in particular, resulting in decreased expression of Trx1. Given the special chemical properties of DATS and the fundamental role of Trx system in tumor progression, DATS could be developed as a promising drug candidate for the prevention of TNBC metastasis.

\section{Materials and Methods}

\section{Reagents and cell culture}

DAS/DADS/DATS/Dipropyl trisulfide (DPTS) (LKT Laboratories) were determined to be $98 \%$ purity by HPLC. Each compound was dissolved in 100\% DMSO. PX-12 was from Cayman Chemical. DL-dithiothreitol (DTT) and Iodoacetic acid (IAA) were obtained from Sigma-Aldrich (St. Louis, USA). $8 \mu \mathrm{m}$ Corning Transwell filter discs (for migration assay) were from Fisher Scientific (Nepean, Ontario, Canada). Actin-Tracker Green (for Immunofluorescence Staining) was purchased from Beyotime Biotechnology (Shanghai, China).

TNBC cell lines MDA-MB-231 and HS 578T were obtained from the Chinese Academy of Sciences Cell Bank of Type Culture Collection (CBTCCCAS, Shanghai, China). MDA-MB-231 cells and HS 578T were tested as being mycoplasma free by using the Lonza MycoAlert Mycoplasma Detection Kit, and authenticated by examination of morphology and consistent in vivo performance. The breast cancer cell lines were cultured in DMEM supplemented with $10 \%$ fetal bovine serum, $100 \mu \mathrm{g} / \mathrm{mL}$ penicillin, and $100 \mu \mathrm{g} / \mathrm{ml}$ streptomycin and were maintained in an incubator with a humidified atmosphere of $95 \%$ air and $5 \% \mathrm{CO}_{2}$ at $37^{\circ} \mathrm{C}$.

\section{Construction of tissue microarray}

A total of 20 paraffin-embedded breast cancer tissues with complete clinical pathological data were collected from November 2010 to December 2013 at Department of General Surgery in Nanjing Medical University Affiliated Cancer Hospital. Tissue microarray (TMA) was constructed as described previously [27]. In brief, duplicates of core tissues (2 $\mathrm{mm}$ in diameter) were obtained from individual donor blocks and arranged in new recipient TMA paraffin blocks using a trephine apparatus [28]. All TMA blocks were confirmed to contain suitable tumor and para-cancer normal tissues via H\&E staining. A total of 20 TMA blocks were created from this patient cohort.

\section{Transwell migration assay}

Cell motility was tested in a Transwell Boyden Chamber (Corning Costar, Cambridge, Massachusetts) using a polycarbonate filter ( $8 \mu \mathrm{m}$ pores). $2 \times 10^{6} \mathrm{MDA}-\mathrm{MB}-231$ cells were resuspended in medium containing various concentrations of DATS and carefully transferred into the upper chambers. The lower chamber was filled with $600 \mu \mathrm{L} \mathrm{20 \%} \mathrm{FBS} \mathrm{medium} \mathrm{to} \mathrm{attract} \mathrm{cells} \mathrm{in} \mathrm{the} \mathrm{upper} \mathrm{chambers.} \mathrm{The} \mathrm{chamber} \mathrm{was} \mathrm{incubated} \mathrm{at}$ $37^{\circ} \mathrm{C}$ for $6 \mathrm{~h}$. The cells on the upper side of the filter membrane were removed by wiping with cotton swabs after gentlely taking out of the filter from the chamber. The filter was fixed with $4 \%$ paraformaldehyde at $4^{\circ} \mathrm{C}$ and stained with $0.1 \%$ crystal violet stain solution. Cells penetrated the pore of the filter were fixed onto a glass slide. Cells in five randomly chosen microscopic fields $(\times 200)$ of the lower slide were counted. Experiments were performed independently three times and the counts were averaged [29].

Thioredoxin redox status assay

Briefly, MDA-MB-231 cells incubated with $20 \mu \mathrm{M}$ DATS for $24 \mathrm{~h}$ were collected with guanidine hydrochloride solution containing the iodoacetic acid (IAA) in $1.5 \mathrm{ml}$ tube. $0.5 \mathrm{mM}$ dithiothreitol (DTT) and $10 \mu \mathrm{M}$ IAA were added and then incubated for $30 \mathrm{~min}$. Ultrafiltration tubes were used for incubation cell lysate desalination at $4^{\circ} \mathrm{C}, 3000 \mathrm{rpm}$ centrifuged for $30 \mathrm{~min}$. Sample buffer without mercaptoethanol was used for protein sample dilution. Protein concentration was quantified using BCA method. The protein samples obtained were subjected to electrophoresis in $12 \%$ non-denaturing acrylamide gels [30]. 


\section{Cellular Physiology Cell Physiol Biochem 2018;50:1945-1963 and Biochemistry Published \begin{tabular}{l|l} 
DOI: 10.1159/000494874 2018 The Author(s). Published by S. Karger AG, Basel \\
(c) 2018 www.karger.com/cpb
\end{tabular}}

Liu et al.: Targeting Trx-1 Limits Metastasis

Thioredoxin reductase activity

TrxR activity was determined in the protein lysate using a commercially available kit, as per the instructions of the manufacturer (Cayman Chemical Company, Ann Arbor, MI, USA). In this assay, TrxR uses NADPH to reduce 5, 5-dithiobis-2-nitrobenzoic acid to 5-thio-2-nitrobenzoic acid. In terms of determining TrxR activity in cells with probe (TrxR probe was generously gifted from Dr. Jianguo Fang from State Key Laboratory of Applied Organic Chemistry in Lanzhou University), TrxR probe was 1000-fold diluted using cell culture medium. All cells were treated polysulfide for $21 \mathrm{~h}$, then TrxR probe was added for $4 \mathrm{~h}$. PBS was used to wash the unloaded probe 2-3 times. The fluorescence intensity was detected in fluorescent microplate reader at $438 / 538 \mathrm{~nm}$ wavelength $[31,32]$.

\section{Western blot assay}

Whole-cell lysates were prepared with RIPA buffer containing protease and phosphatase inhibitors. Nuclear and cytoplasmic cell extracts were prepared using the NE-PER Nuclear and Cytoplasmic Extraction kit (Thermo, Rockford, USA). Equal amounts of cell lysates (50 $\mu \mathrm{g})$ were loaded on 8 or $10 \%$ SDS-PAGE and transferred onto PVDF membranes. After the membranes were blocked, they were incubated with indicated primary antibodies including phosphor-ERK, ERK (1:1000, Cell Signaling Technology), Trx-1 (1:10000, Abcam), TrxR (1:20000, Abcam), TXNIP (1:1000, Abcam), GPADH (1:5000, Bioworld Technology), followed by incubation with horseradish peroxidase-conjugated IgGs (1:10000, Bioworld Biotechnology). Target proteins were developed with an ECL detection agent (Millipore, Braunschweig, Germany).

\section{Immunofluorescence}

Cells were fixed in $4 \%$ paraformaldehyde for $30 \mathrm{~min}$, permeabilized in phosphate-buffered saline $0.1 \%$ Triton-X 100 for $10 \mathrm{~min}$, and blocked with 3\% bovine serum albumin for $1 \mathrm{~h}$ at room temperature. The cells were then incubated overnight with the appropriate antibodies, followed by incubation for $1 \mathrm{~h}$ at room temperature with secondary fluorescent antibodies. Finally, to label nuclei, cells were counterstained with DAPI for 5 min and then observed using a confocal microscope (Leica, Wetzlar, Germany).

\section{Animal studies}

6 7-week-old BALB/c female nude mice (16-20 g) were obtained from Shanghai Laboratory Animal Center, Chinese Academy of Sciences. Animal welfare and experimental procedures were performed strictly in accordance with the institutional guidelines and approved by the Animal Care and Ethics Committee of the University of the Nanjing University of Chinese Medicine. The spontaneous metastatic animal model was established according to methods described previously [33]. $4 \times 10^{6}$ MDA-MB-231 cells in $200 \mu$ PBS were injected into the right breast pads of BALB/c nude mice. Tumor volume was grown to $100 \mathrm{~mm}^{3} 9$ days following injection, DATS were given to tumor-bearing mice by daily oral administration at doses of 25, 50 $\mathrm{mg} / \mathrm{kg}$ from day 9 to day 40. The control group received olive oil only. Tumor-bearing animals were weighed once every 3 days. Tumor size was determined once per 3 days by caliper and volume was calculated according to the formula: volume $=$ width $^{2} \times$ length $\times 0.5$. Mice were sacrificed at 23 days post excision of primary tumors. Lungs and livers were removed, and fixed. Metastatic colonies were counted under a dissecting microscope. Lungs and livers were photographed to show tumor spontaneous metastasis.

The experimental metastasis was established by the methods described previously [34]. MDA-MB-231 cells $\left(2 \times 10^{7}\right.$ cells $/ \mathrm{ml}$ in $\left.0.2 \mathrm{ml} \mathrm{PBS}\right)$ were injected into BALB/c nude mice via the lateral tail vein. Following the MDA-MB-231 cell injection, mice were randomly divided into different treated groups. DATS were given by daily oral administration at doses of $25,50 \mathrm{mg} / \mathrm{kg}$ from day 3 . Control animals received daily oral administration of olive oil. Animals were weighed every 3 days. All mice were sacrificed at 23 days following tumor injection. Lungs and livers were removed and fixed. Metastatic foci on the surface of lung and liver were photographed.

\section{Statistical analysis}

The results were analyzed by two-tailed student t-test SD using SPSS 11.0 (Aspire Software International, Leesburg, VA) and P-values were calculated. The difference was considered significant between two samples if $\mathrm{P}<0.05$. 


\section{Results}

Trx-1 is overexpressed in human breast cancer

We initially performed immunohistochemistry of Trx-1 in human breast cancer tissues and corresponding para-cancer normal tissues from 20 patients. Analysis of staining results indicated that Trx-1 expression was significantly higher in breast cancer tissues than in canceradjacent tissues (Fig. 1A). In order to better analyze patient data, samples were divided into four groups, including non-TNBC without invasion (\#1 and \#2), TNBC without invasion (\#3 and \#4), non-TNBC with invasion (\#5 and \#6) and TNBC with invasion (\#7 and \#8), according to whether or not there was invasiveness and if the patient belonged to TNBC. As shown in Fig. 1B, invasive breast cancer (\#5 and \#6) accompanied by increased level of Trx-1 compared to non-invasive cancer (\#1 and \#2) and TNBC with invasion (\#7 and \#8) displayed the most highly expressed Trx-1 among all patient samples. of note, nuclear expression of $\operatorname{Trx}$ was detected more frequently in TNBC with invasion group than the other groups.

Tissue staining was then scored on the basis of the intensity of Trx-1 labeling and percentage of Trx-1-positive tumor cells. Relational analysis between Trx-1 levels and lymph node metastasis, invasion or ER/PR/HER-2 expression was accordingly examined. As illustrated in Table 1, the expression of Trx-1 was positively correlated with lymph node metastasis $(p<0.05)$ rather than tumor invasion and ER/PR/HER-2 expression. Increased levels of Trx-1 in parallel with more positive nuclear staining were observed in breast cancer with lymph node metastasis (Fig. 1C). Furthermore, we used high- (MDA-MB-231 and Hs578T) and low-/non- (MCF-7 and ZR-75-1) metastasis cell lines of human breast cancer as an experimental model to compare their expression levels for Trx-1. Western blot data showed that two breast cancer cell lines with high metastatic potential, MDA-MB-231 and Hs578T, presented strong expression of Trx-1, whereas low metastatic cell lines (MCF-7 and ZR-75-1) were associated with its weak expression (Fig. 1D), which were consistent with the results of tissue staining observed in patient samples.

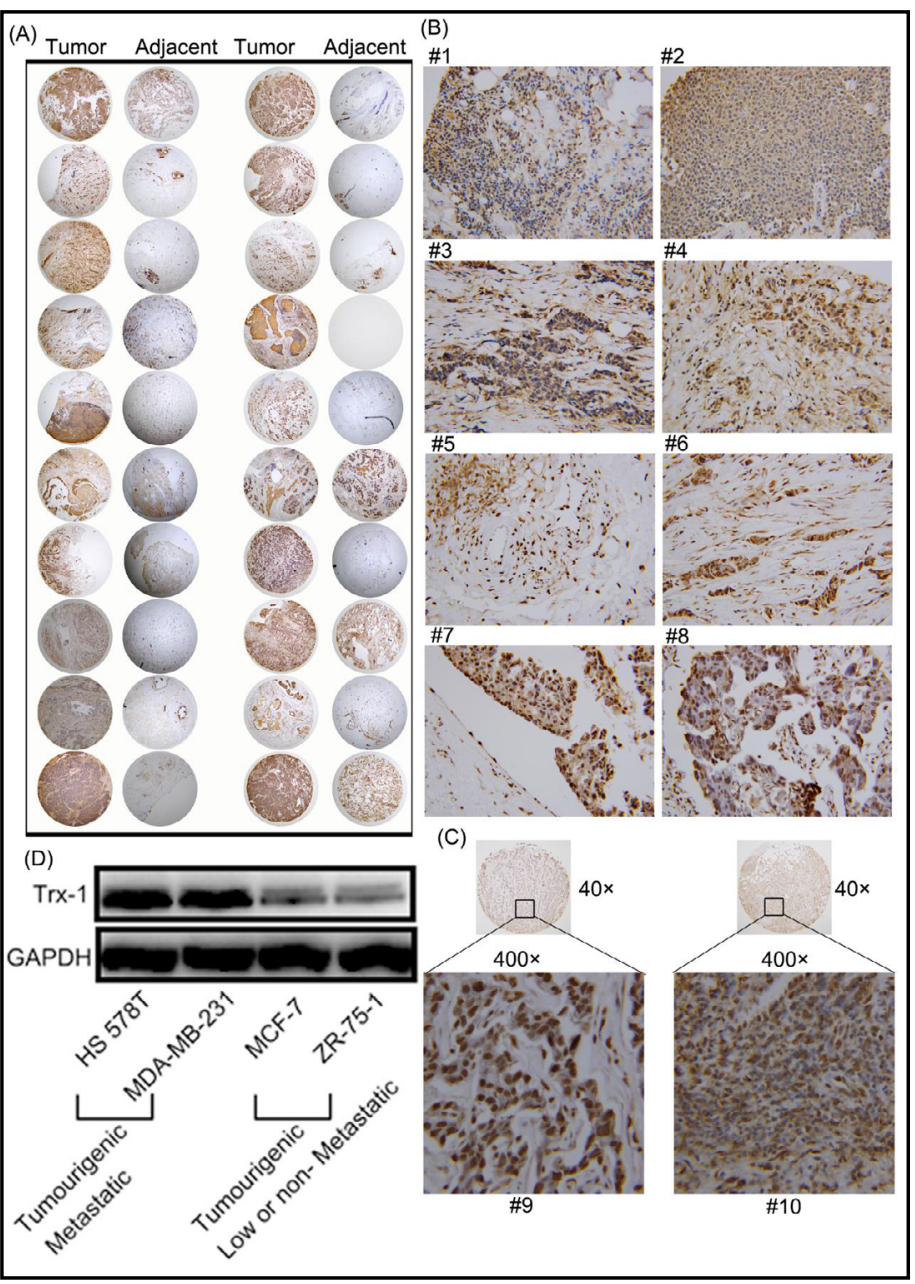

Fig. 1. Trx-1 is overexpressed in human breast cancer. (A) Immunohistochemistry of Trx-1 on tissue microarrays containing breast cancer tissues and cancer-adjacent tissues. Images of representative staining from 20 patients with breast cancer are shown. (B) Representative immunohistochemistrical images from $\# 2$ ), TNBC without invasion (\#3 and \#4), non-TNBC with invasion (\#5 and \#6) and TNBC with invasion (\#7 and \#8). (C) Representative immunohistochemistrical images of breast cancer samples from patients with (\#9) or without (\#10) lymph node metastasis are shown. (D) Protein expression of Trx-1 in high- (MDA-MB-231 and Hs578T) and low-/non- (MCF-7 and ZR-75-1) metastasis cell lines of human breast cancer, GAPDH was used as a loading control. 


\section{Cellular Physiology Cell Physiol Biochem 2018;50:1945-1963

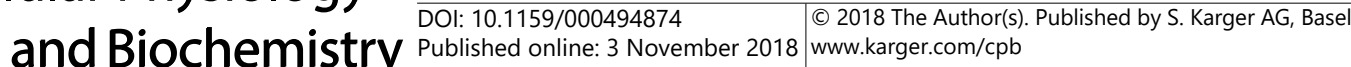

Liu et al.: Targeting Trx-1 Limits Metastasis

Inhibition of thioredoxin system resulted in diminished metastatic potential of breast cancer cells

Given the striking role of Trx system in the breast cancer progression, we intended to investigate whether the inhibition of Trx1 as the predominant molecule in Trx system was associated with reduced malignancy. To this end, PX-12, an irreversible Trx-1 inhibitor [35] whose structure contains two sulphur atoms (Fig. 2A), was used to treat MDA-MB-231 breast cancer cells. It was observed that PX-12 (from 4 to 16 $\mu \mathrm{M}$ ) dose-dependently inhibited tumor cell proliferation (Fig. 2B) and markedly lowered Trx-1 protein expression at $4 \mu \mathrm{M}$ (Fig. 2C). Therefore, in the following experiments, the dose of PX-12 used was $4 \mu \mathrm{M}$. To determine the role of PX-12 in cell contact, colony-scattering assay was carried out to assess the capability of cells to detached from colonies. Colonies with different morphologies were generally divided into three categories: compact, loose and scattered. The data showed that PX-12 lead to more compact colonies and fewer scattered colonies (Fig. 2D) and morphology images displayed that PX-12 treated cells were inclined to be round shape and less pseudopodia compared to non-treated cells (Fig. 2E), indicating the metastatic property of MDA-MB-231 breast cancer cells was remarkably attenuated. Further, $4 \mu \mathrm{M}$ of PX-12 dramatically inhibited the migration (Fig. 2F and 2G) and invasion of MDA-MB-231 cells (Fig. 2H and 2I), as defined by wound healing assay and transwell cell invasion assay.

\section{DATS modulates the thioredoxin system in breast cancer}

Since Trx-1 inhibition appeared to be a reliable strategy for the prevention of tumor metastasis, we then explored whether DATS was able to target Trx system. Firstly, computational docking was employed to predict bound conformations and free energies of binding for DATS to a variety of thioredoxin-related molecules, such as oxidized Trx-1, reduced Trx-1, Trx-2, TXNIP and TrxR. Interestingly, docking data demonstrated that DATS showed potential binding affinities with these molecules (negative values of energies) and docking scores ranged from -22.79 to $-6.09 \mathrm{kcal} / \mathrm{mol}$ (Fig. 3C). DATS exhibited high affinities to Trx-1 and TrxR as the binding required relatively low energies and reduced Trx-1 was predicted to be the potential target due to its lowest docking sore (Fig. 3A and 3B).

In order to confirm the computational results, MDA-MB-231 and Hs578T high metastatic breast cancer cell lines with abundant Trx-1 expression were chosen. It was found that DATS significantly attenuated Trx-1 levels in MDA-MB-231 (5-20 $\mu \mathrm{M})$ and in Hs578T $(10-20 \mu \mathrm{M})$ cells. The expression of Trx-1 was also dropped in a time-dependent manner from $12 \mathrm{~h}$ treatment by $10 \mu \mathrm{M}$ DATS (Fig. 4A and 4B). Moreover, DATS contributed to elevated levels of TXNIP, which is the endogenous inhibitor of Trx-1, in dose- and time- dependent manners in both cell lines. However, no obvious changes were observed in terms of TrxR expression after the treatments of DATS (Fig. 4A and 4B). Due to the fact that nuclear expression of Trx- 1 is likely to control the expression of downstream transcription factors (NF- $\kappa B$, AP1 , etc), immunofluorescent staining was used to assess its distribution within the cells. As demonstrated in Fig. 4C, Trx-1 nuclear expression in MDA-MB-231 cells was sharply reduced by DATS compared to control cells in which Trx-1 was localized both nucleus and cytosol. Consistently, $20 \mu \mathrm{M}$ of DATS resulted in decreased Trx-1 nuclear expression in Hs578T cells where Trx-1 mainly located in nucleus (Fig. 4D).

Considering that reduced Trx-1 played a key role in its translocation and had highest affinity with DATS based on computational docking, we further investigated whether DATS had any impacts on distinct forms of Trx-1. To address this, IAA and DTT were used to allow separation of oxidized and reduced forms of Trix-1, respectively. Purified Trx- 1 was reduced with DTT and oxidized with IAA, as visualized in Fig. 5A. Notably, DATS was able to diminish reduced Trx-1 by itself and reverse DTT-mediated reduced form of Trx-1 (Fig. 4E), which was consistent with reduced nuclear translocation. Also, DTT-induced migration of MDA-MB-231 cells was abrogated following the treatment of $20 \mu \mathrm{M}$ DATS and additive inhibitory effect 
Fig. 2. Inhibition of thioredoxin system resulted in diminished metastatic potential of breast cancer cells. (A) Chemical structure of an irreversible Trx1 inhibitor, PX-12. (B) Proliferation of MDAMB-231 cells treated with PX-12 (0-16 $\mu \mathrm{M})$ was evaluated by MTT assay. Data were presented as means \pm SEM. **, $\quad \mathrm{P}<0.01 ; * * *$, $\mathrm{P}<0.001$, paired t-test. (C) Protein expression of Trx-1 following the treatment of PX-12 (0-4 $\mu \mathrm{M})$, GAPDH was used as a loading control. (D) Colony scatter assay was used to describe the dispersion (compact, loose or scatter morphology) of compact colonies of MDA-MB-231 cells treated with $4 \mu \mathrm{M}$ of PX-12. (E) Im mun ofluorescent staining of F-actin (red) and DAPI (blue) for MDA-MB-231 breast cancer cells was performed to determine the cell morphology. (F) Representative images from a timelapse sequence $(0$ and $24 \mathrm{~h}$ ) of MDA-MB-231 cells migrating to heal a wound scratched in a monolayer. (G) Analysis of relative horizontal migration rate of MDA-MB-231 cells treated with $4 \mu \mathrm{M}$ of PX-12 compared to untreated cells. Data were presented as means \pm SEM from three independent experiments. $*, \mathrm{P}<0.05$, paired t-test. $(\mathrm{H})$ Representative images of MDA-MB-231 cell invasion in the absence and presence of $4 \mu \mathrm{M}$ of PX-12 by crystal violet staining. (I) Quantification of the number of invasive MDA-MB-231 cells following the treatment of $4 \mu \mathrm{M}$ of PX-12. Data were presented as means \pm SEM from three independent experiments. $* *, \mathrm{P}<0.01$, paired t-test.

of IAA on cell migration was observed in combination with DATS (Fig. 5B and 5C, Fig. 4F), indicating reduced form of Trx- 1 that targeted by DATS is responsible for metastatic potential of breast cancer cells. It is known that the conversion of Trx- 1 from oxidized to reduced form is dependent on TrxR that is recognized as the only enzyme to catalyze the reduction of Trx1. Thus, the TrxR activity was measured at time intervals using the DTNB (5, 5'- dithiobis (2-nitrobenzoic)) acid assay. It was found that DAT started to exhibit inhibitory effect on TrxR activity from $20 \mu \mathrm{M}$ and remarkably decreased the activity at $40 \mu \mathrm{M}$ in MDA-MB-231 breast cancer cells, which was much obvious than DAS, DADS and DPTS (Fig. 4G). Similarly and more precisely, the evaluation of TrxR activity with its first fluorescent probe revealed that TrxR activity could be measured based on fluorescence and $20 \mu \mathrm{M}$ DATS contributed to the most drop of TrxR activity compared to the same concentrations of DAS, DADS and DPTS (Fig. 4H). 
DATS limited experimental metastasis of human breast carcinoma cells

To investigate whether DATS could play a critical role in preventing breast cancer metastasis in vivo, MDAMB-231 breast cancer cells were intravenously injected into nude mice to establish the experimental metastasis model. As indicated in Fig. 6A, obvious and cauliflower-like metastatic nodules were visualized on the surface of lung and liver harvested from the mice of model group 27 days following the injection of MDA-MB-231 cells. Surprisingly, 25 and 50 $\mathrm{mg} / \mathrm{kg}$ of DATS remarkably diminished the number of metastatic nodules and relieved the lesion of liver and lung caused by metastasis. Moreover, in the model group, H\&E staining revealed that there was large area of metastatic tumor cells in the lung tissue section. In contract, the metastatic area of the lung tissue was dramatically decreased following the administration of 25 and $50 \mathrm{mg} / \mathrm{kg}$ DAT (Fig. 6B and 6C). Of note, there existed observable loss of body weight at the late stage of experimental metastasis model (from day 21 to 27), suggesting severe cachexia occurred. Nevertheless, the delivery of DATS, to a large extent, alleviated the malignancy by preventing weight loss (Fig. 6D). Given the inhibitory effect of DTAS on Trx-1 expression in vitro, we further detected the level of Trx-1 in metastatic nodules of the lung tissue. Immunohistochemistry results demonstrated that DATS dose-dependently reduced the expression Trx-1 in vivo (Fig. 6E and 6F). In addition, Trx-1 nuclear expression was also reduced by DATS (Fig. 6G). Because $\mathrm{NF}-\kappa \mathrm{B}$ is the transcription factor that regulated by Trx- 1 and directly targets MMP2/9 that exert key effects on tumor cell spread, the levels of NF- $\kappa B$ and MMP2/9 were examined as well. It was observed that the expression of NF- $\kappa B$ and MMP2/9 enhanced with the presence of metastatic nodules, whereas $50 \mathrm{mg} / \mathrm{kg}$ DATS strikingly attenuated the brown staining of MMP2 $/ 9$ and NF- $\mathrm{KB}$ level could be significantly reduced by DATS from $25 \mathrm{mg} / \mathrm{kg}$ (Fig. $6 \mathrm{H}$ and 6I).

\section{DATS suppressed spontaneous metastasis of primary mammary fat pad tumors}

In order to develop a system that models the entire metastatic process, MDA-MB-231 breastcarcinoma cellswere orthotopically into the mammary fatpads of immunocompromised mice to evaluate the role of DATS in spontaneous metastasis. Mice were monitored for the development of primary xenograft tumors and sacrificed 33 days following the cell injection. As depicted in Fig. 7A and 7B, tumor volume during the process of development and tumor weight on the final day did not significantly differ between model and DATS-treated groups though slightly reduction was observed following the administration of DATS. However, the overall metastatic tumor burden in the target organs lung and liver was significantly lower in DATS-treated groups compared to model control group. $50 \mathrm{mg} / \mathrm{kg}$ of DATS nearly achieved similar inhibition of macrometastasis on the surface of lung and liver as $5 \mathrm{mg} / \mathrm{kg}$ cisplatin (DDP) (Fig. 7C). In addition, H\&E staining of lung tissue uncovered that the micrometastatic area was dramatically decreased after the treatment of 25 and $50 \mathrm{mg} / \mathrm{kg}$ of DATS (Fig. 7D and 7E). Despite DDP contributed to significant drops in terms of tumor growth and metastasis, it also caused subsequent weight loss, which could, to some extent, be avoided by DTAS (Fig. 7F).

Similarly to experimental metastasis model, harvested primary mammary fat pad tumor tissue was immunohistochemically stained for Trx-1, MMP2/9 and NF- $\kappa$ B. As demonstrated 
Fig. 4. DATS modulates the thi oredoxin system in breast cancer. (A) Protein expression of Trx-1, TXNIP and TrxR in M D A - M B - 231 cell lysates after the treatment of DATS. GAPDH was used as a loading control. Changes in the levels of Trx-1, TXNIP and TrxR in MDA-MB-231 cell lysates were measured as pixel density and normalized to GAPDH. Data were presented as means \pm SEM from three independent experiments. *, $\quad \mathrm{P}<0.05$; **, $\quad \mathrm{P}<0.01$, paired t-test. (B) Protein expression of Trx-1, TXNIP and TrxR in Hs578T cell lysates after the treatment of DATS. GAPDH was used as a loading control. Changes in the
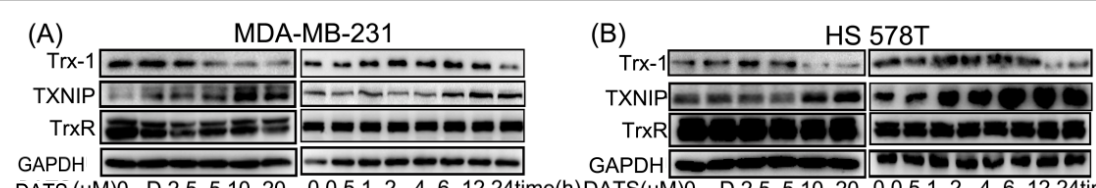

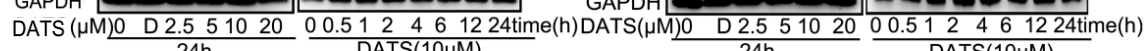
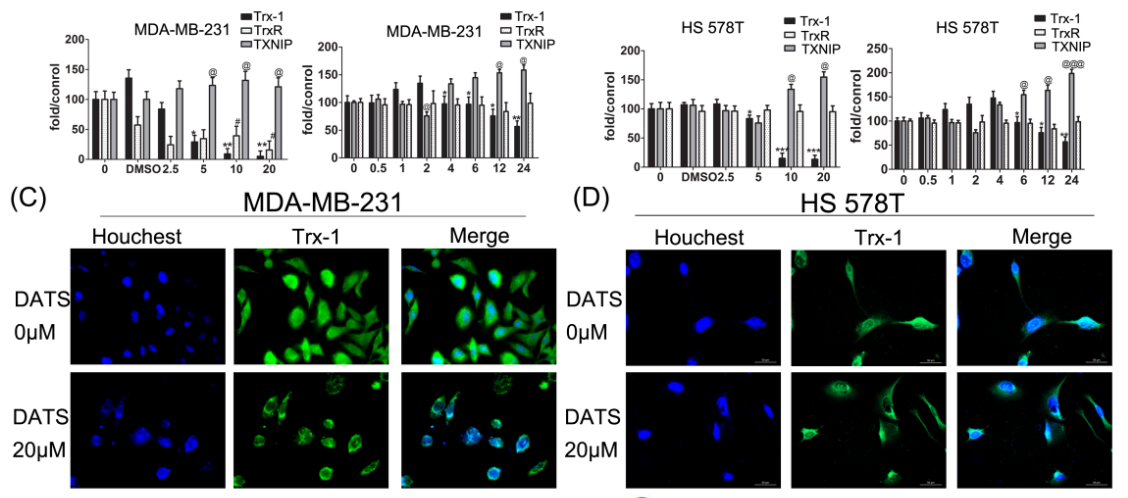

(E)

(F)
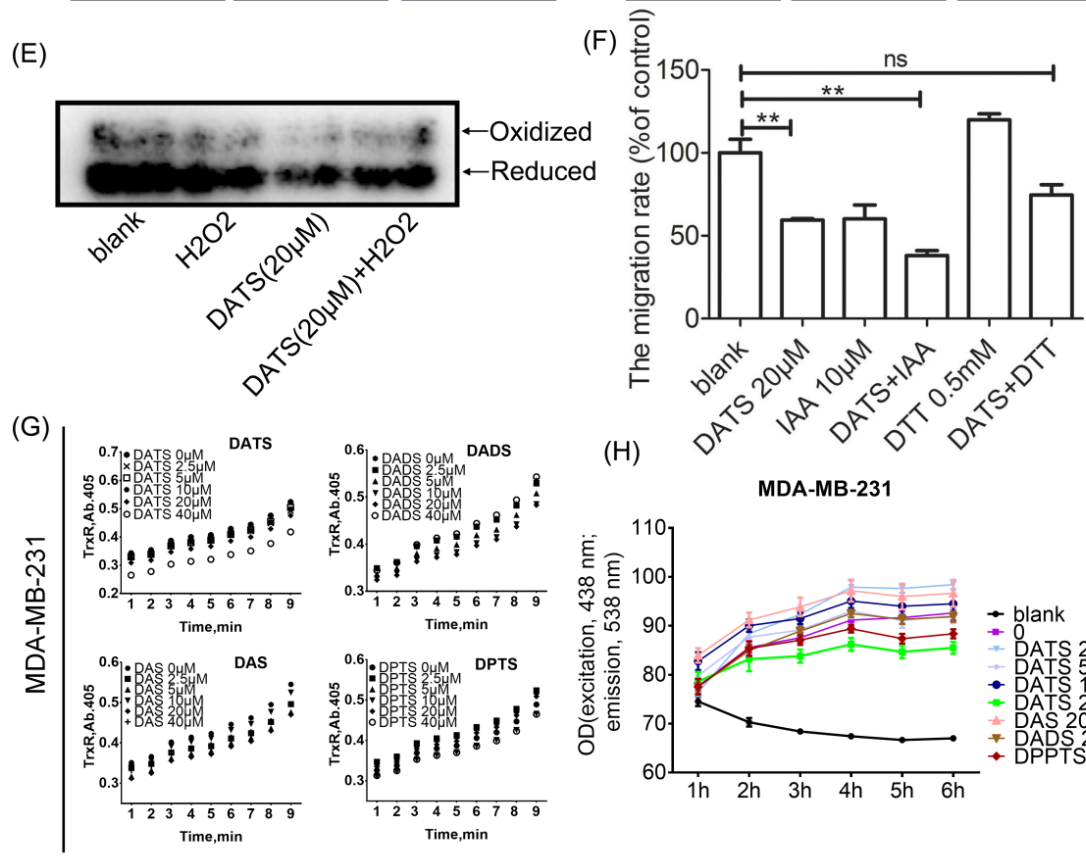

$(\mathrm{H})$

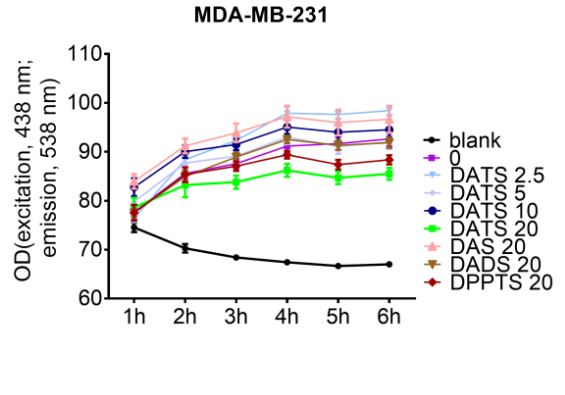
levels of Trx-1,

TXNIP and TrxR in Hs578T cell lysates were measured as pixel density and normalized to GAPDH. Data were presented as means \pm SEM from three independent experiments. ${ }^{*}, \mathrm{P}<0.05 ;{ }^{* *}, \mathrm{P}<0.01$; ${ }^{* * *}, \mathrm{P}<0.001$, paired t-test. (C) Immunofluorescent staining of Trx-1 (green) and DAPI (blue) was performed to evaluate Trx-1 translocation in the absence and presence of DATS in the MDA-MB-231 cells. (D) Immunofluorescent staining of Trx-1 (green) and DAPI (blue) was performed to evaluate Trx-1 translocation in the absence and presence of DATS in the Hs578T cell. (E) Expression of oxidized and reduced Trx-1 in the MDA-MB-231 cell lysates after the treatment of $20 \mu \mathrm{M}$ DATS was shown by Western Blot. (F) The migration of MDA-MB-231 treated with IAA, DTT, and DATS, ${ }^{* *}, \mathrm{P}<0.01$. (G) TrxR activity following treatment of different concentrations of DATS was measured at time intervals using the DTNB acid assay. (H) TrxR activity following treatment of different concentrations of DATS was assessed by fluorescent probe.

in Fig. 7G, MMP2 and MMP9 were highly expressed in the primary mammary fat pad tumors, whereas treatment of DATS at $50 \mathrm{mg} / \mathrm{kg}$ significantly diminished their expression. Notably, in model group, NF- $\mathrm{NB}$ expression was strikingly elevated and frequently detected in nucleus, which could be rescued in the presence of DTAS (Fig. 7G). More importantly, DATS also dosedependently resulted in significant decrease in Trx-1 protein levels in primary tumors, as 


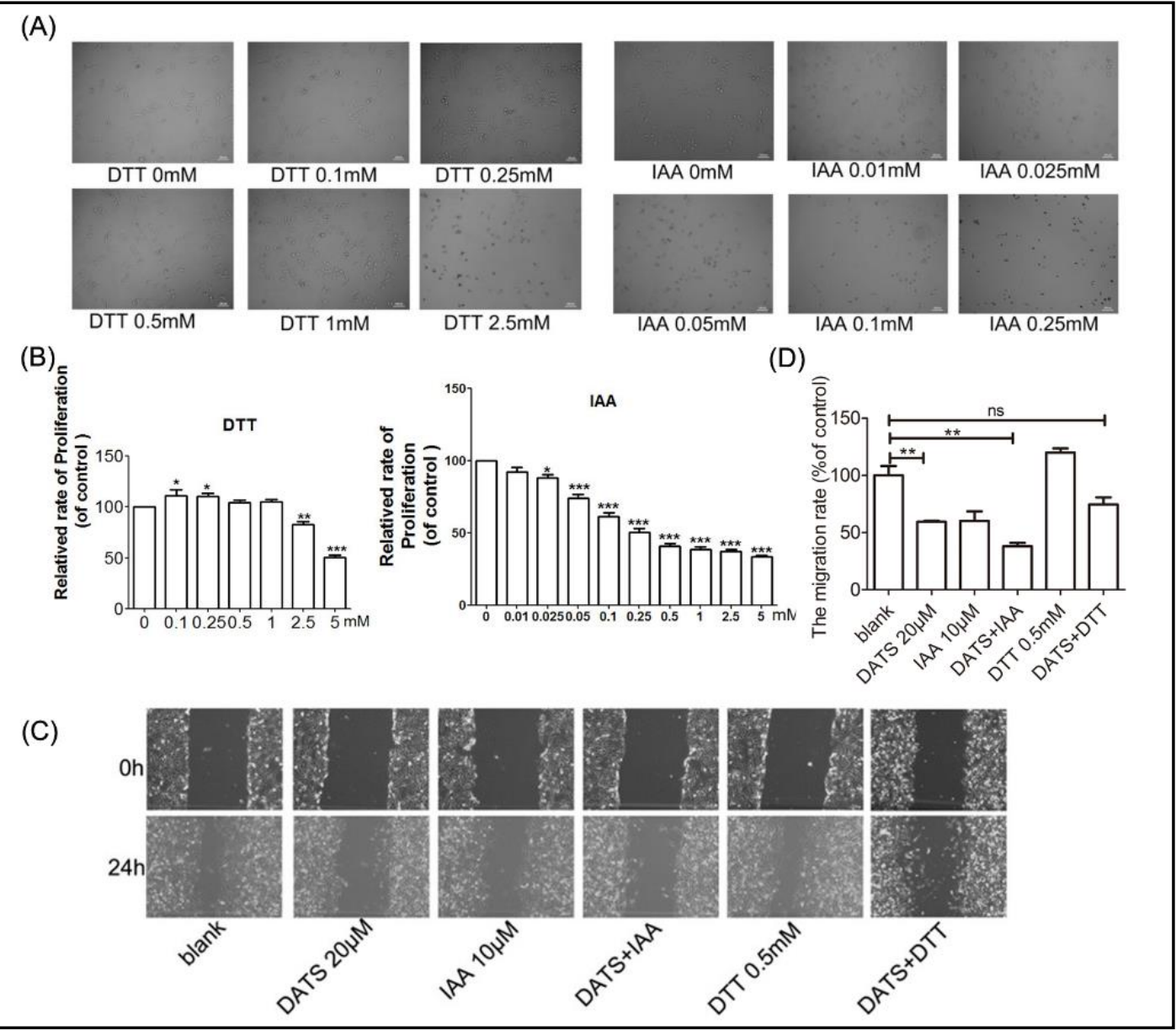

Fig. 5. The effects of DTT and IAA on MDA-MB-231 breast cancer cells. (A and B) An in vitro study was initiated by treating MDA-MB-231 cells with increasing doses of DTT ( 0 to $5 \mathrm{mM}$ ) and IAA (0 to $5 \mathrm{mM}$ ) for $24 \mathrm{~h}$. The viability of the drug-treated cells was measured using the MTT assay. Results were expressed as a percentage of control, which was considered as $100 \%$. Data were presented as mean \pm SEM and at least three separate experiments were performed. Cellular morphological changes of MDA-MB-231 breast cancer cells. (C) Confluent MDA-MB-231 cells were scratched and incubated with $0.5 \mathrm{mM}$ DTT, $10 \mu \mathrm{M}$ IAA respectively and together with $20 \mu \mathrm{M}$ DATS. The area covered by migrating cells was recorded by phase-contrast microscopy connected to a digital camera at time 0 and 24h. (D) The wound closure area was calculated by measuring the diminution of the wound bed surface upon time using Image J software. Representative pictures of three independent experiments were shown. ${ }^{* *}, \mathrm{P}<0.01$.

defined by both immunohistochemistry and western blot (Fig. 8A and 8B). Consistently, in view of lung tissue as the metastatic prevalent organ, the levels of MMP2/9, NF- $\kappa B$ and Trx-1 were all downregulated after the administration of DATS (Fig. 9A).

The effect of DATS on breast caner metastasis was mediated by Trx-1

Since DATS exhibited high affinity to Trx-1 and was able to lead to dramatic decrease of its expression, we further investigated whether the effects of DATS on breast cancer cells were mediated by Trx-1. In order to answer this question, western blot was initially utilized to select the most efficient siRNA (\#345) in which suitable design caused a more efficient silencing than the other two siRNAs (\#235 and \#435) (Fig. 10A). Surprisingly, as demonstrated in Fig. 10B, the enhanced expression of TXNIP by $20 \mu \mathrm{M}$ DATS was clearly rescued following the transfection of Trx-1 siRNA into MDA-MB-231 cells though there was no obvious change was observed in TrxR expression between control and knockdown groups in the presence of DATS. Furthermore, transwell migration assay showed that the 
Fig. 6. DATS limited experimental metastasis of human breast carcinoma cells. (A) Representative pictures show the reduction in number of experimental metastatic foci in lungs and livers after the treatment of DATS. (1) Normal group; (2) Model group; (3) $25 \mathrm{mg} / \mathrm{kg}$ DATS treated group; (4) $50 \mathrm{mg} / \mathrm{kg}$ DATS treated group. (B) Lung metastasis was quantified as percentage of metastatic area in the lung. Data were presented as means \pm SEM from three independent experiments. *, $\mathrm{P}<0.05$; **, $\mathrm{P}<0.01$, paired t-test. (C) $\mathrm{H} \& \mathrm{E}$ stain histological analysis of lung sections indicate experimental lung metastasis was inhibited by $25 \mathrm{mg} /$ $\mathrm{kg}$ and $50 \mathrm{mg} / \mathrm{kg}$ DATS. (D) Mean body weight of mice receiving $25 \mathrm{mg} / \mathrm{kg}$ and 50 $\mathrm{mg} / \mathrm{kg}$ DATS. Data represents mean \pm SEM, paired t-test. (E) Immunohistochemical staining for $\operatorname{Trx}-1$ in the absence and presence of DATS $(25 \mathrm{mg} / \mathrm{kg}$ and $50 \mathrm{mg} / \mathrm{kg}$ ) in normal and metastatic lung tissues. (F) Quantification of histochemical staining for Trx-1. Data were presented as means \pm SEM. *, $\mathrm{P}<0.05$, paired t-test. (G) The nuclear expression of Trx-1 in lung tissue. (H) Immunohistochemical staining for MMP-2, MMP-9 and the NF$\kappa \mathrm{B}$ subunit p65 in the absence and presence of DATS $(25 \mathrm{mg} /$ $\mathrm{kg}$ and $50 \mathrm{mg} / \mathrm{kg}$ ) in normal and metastatic lung tissues. (I) Quantification of histochemical staining for MMP-2, MMP-9 and $\mathrm{NF}-\kappa \mathrm{B}$. Data were presented as means \pm SEM. *, $\mathrm{P}<0.05$, paired t-test.
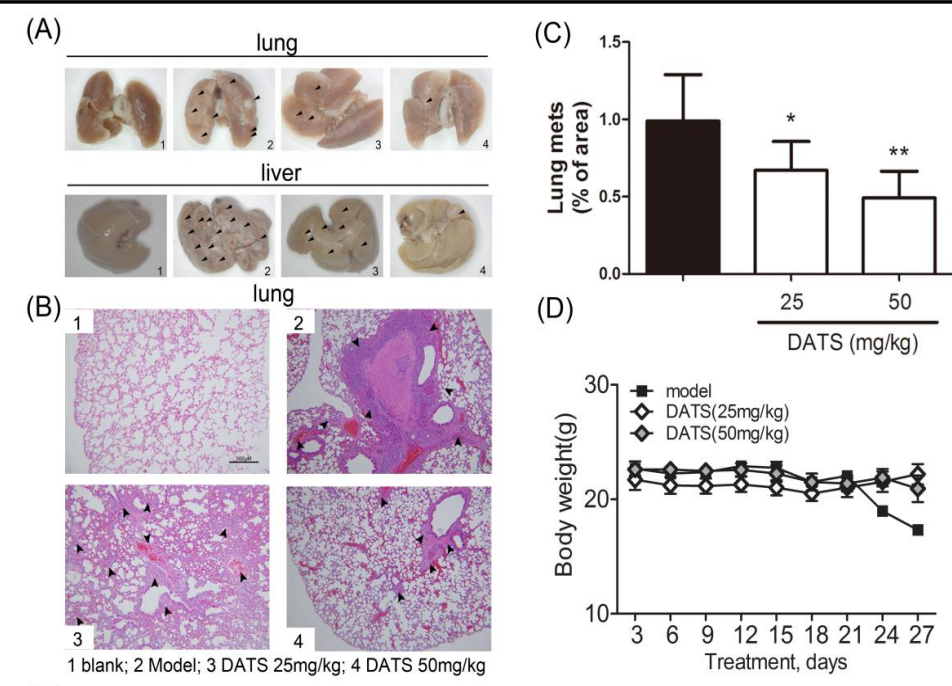

(E)
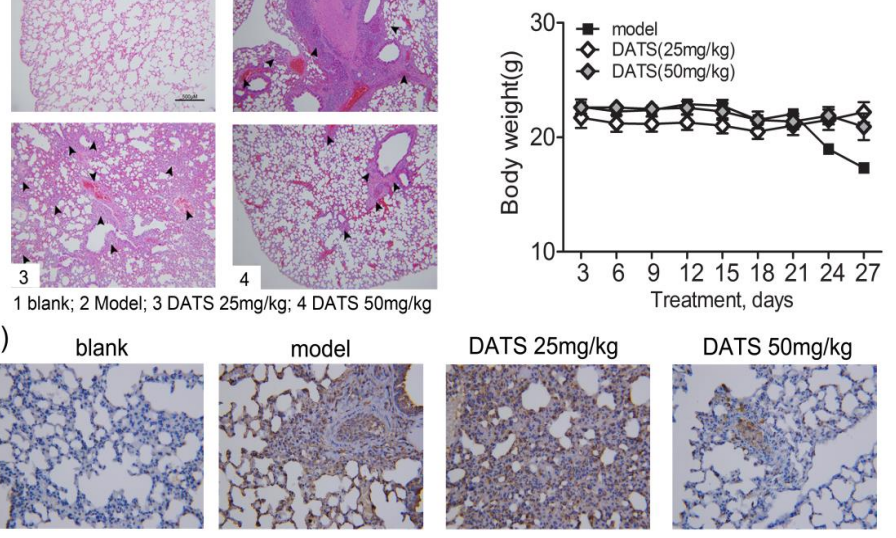

(F)
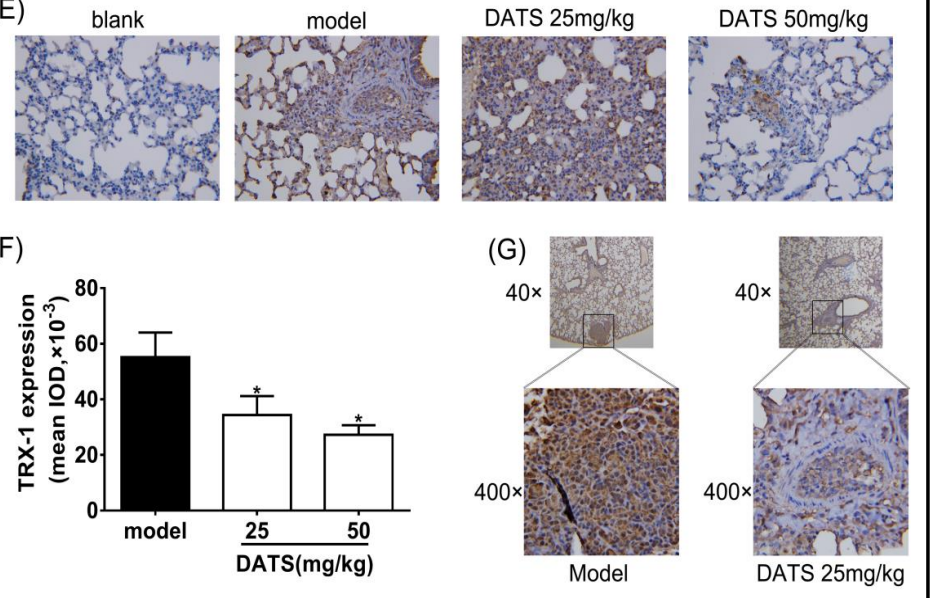

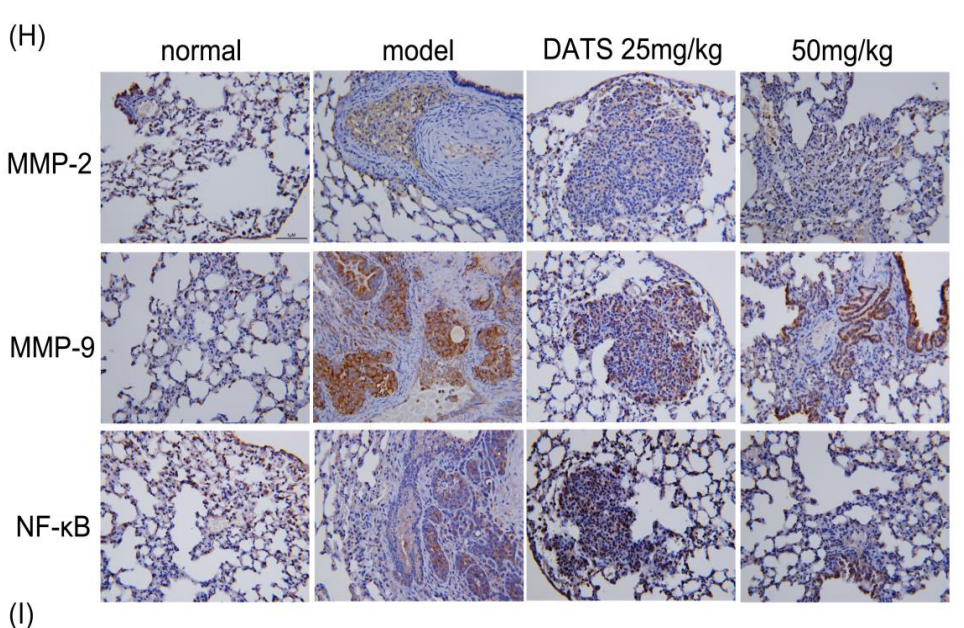

(I)
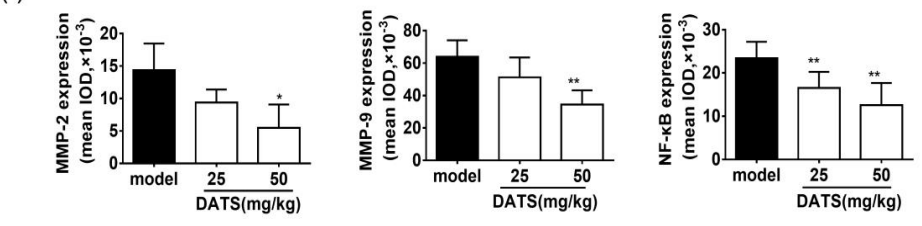
Fig. 7. DATS

s u p p r e s s ed spontaneous $\mathrm{m}$ e $\mathrm{t}$ a s $\mathrm{t}$ a $\mathrm{s}$ is of primary mammary fat pad tumors. (A) Growth curve of M D A - M B - 231 breast carcinoma given $25 \mathrm{mg} /$ $\mathrm{kg}, \quad 50 \mathrm{mg} / \mathrm{kg}$ DATS or $5 \mathrm{mg} /$ $\mathrm{kg}$ of DDP into nude mice. Data represents mean \pm SEM. ${ }^{* *}, \mathrm{P}<0.01$, paired t-test. (B) Weight of MDAMB-231 breast tumors treated by $25 \mathrm{mg} / \mathrm{kg}, 50$ $\mathrm{mg} / \mathrm{kg}$ DATS or 5 $\mathrm{mg} / \mathrm{kg}$ of DDP 33 days following the tumor cell injection. Data represents mean \pm SEM. * $\mathrm{P}<0.05$, paired t-test. (C) Representative pictures show the reduction in number of spontaneous metastatic foci in lungs and livers after the treatment of DATS. (1) Normal group; (2) Model group; (3) $25 \mathrm{mg} / \mathrm{kg}$ DATS treated group; (4) $50 \mathrm{mg} / \mathrm{kg}$ DATS treated group; (5) $5 \mathrm{mg} / \mathrm{kg}$ DDP treated group. (D) H\&E stain histological analysis of lung sections indicate spontaneous lung metastasis was inhibited by $25 \mathrm{mg} / \mathrm{kg}$ and $50 \mathrm{mg} / \mathrm{kg}$ DATS. (E) Lung metastasis was quantified as percentage of metastatic area in the lung. Data were presented as means \pm SEM from three independent experiments. *, $\mathrm{P}<0.05$; ${ }^{* *}$, $\mathrm{P}<0.01$, paired t-test. (F) Mean body weight of mice with different treatments.

suppression effect of DATS on MDA-MB-231 cell migration was abrogated following the silence of Trx- 1 despite significant reduction could be visualized by Trx-1 siRNA compared to control siRNA (Fig. 10C and 10D).

Given the fact that NF- $\mathrm{KB}$ was the key transcription factor that could be modulated by DATS in primary tumor and lung tissue, we then determined whether Trx-1 participated into the inhibitory effect. We thus examined the expression of a series of metastasis-associated proteins that potentially were regulated by Trx-1. It was found that DATS could not achieve further decrease in ERK phosphorylation on top of Trx-1 siRNA (Fig. 10E). Similarly, the produced difference in the expression of RhoA and Rac1/2/3 by DATS was reduced following the silence of Trx-1. Moreover, the inhibitory effects of DATS on phosphorylation of FAK at Tyr397 and Tyr925 and expression of MMP2/9 were all reversed by Trx-1 siRNA (Fig. 10F), indicating the functions were mediated by Trx-1 expression. Interestingly, DATS showed no obvious effect on the expression of FAK that is mainly associated with adhesion (Fig. 10F), suggesting that there was no clear link between FAK level and attenuated Trx-1 by DATS. 


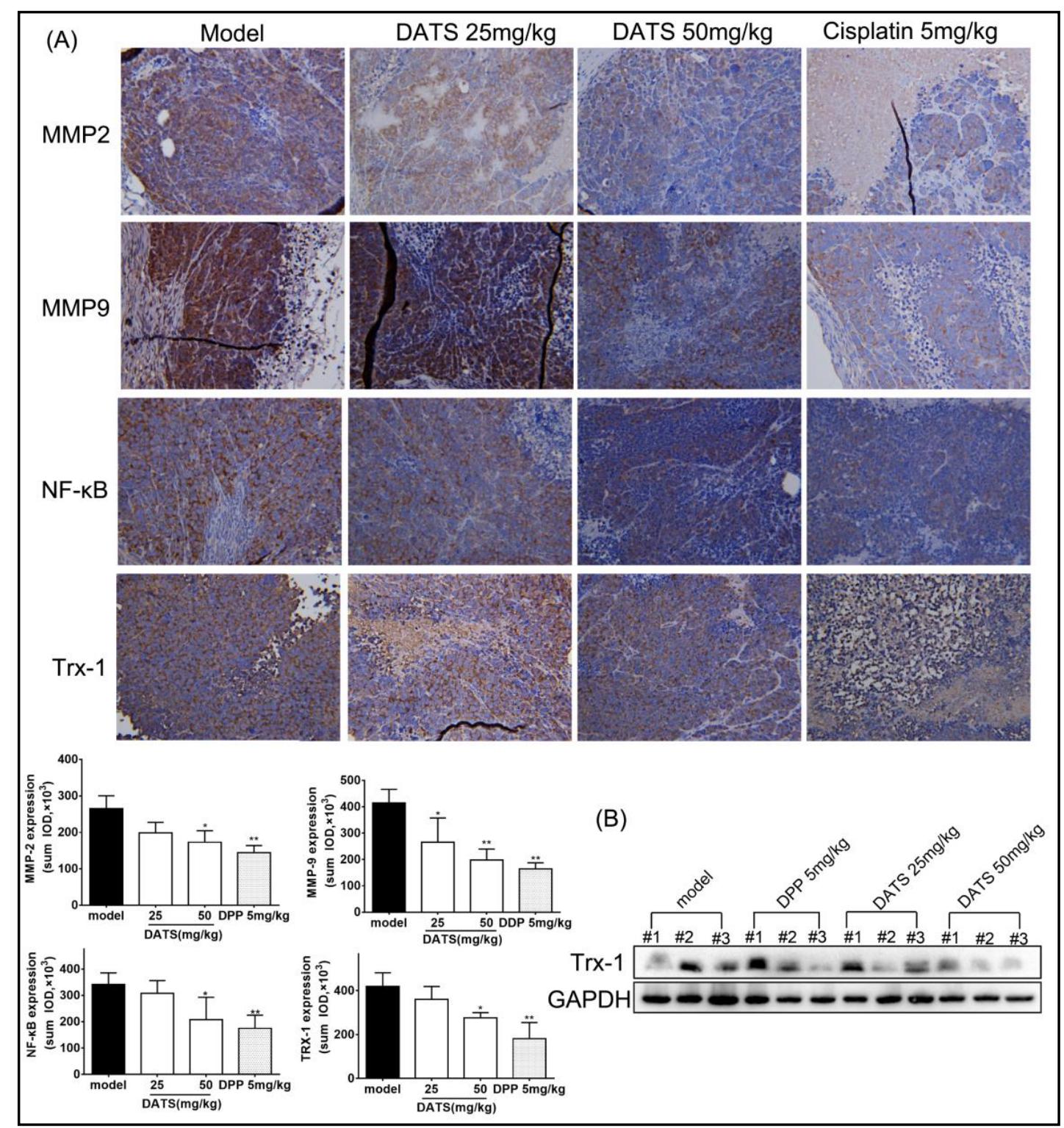

Fig. 8. DATS suppressed spontaneous metastasis of primary mammary fat pad tumors. (A) Immunohistochemical staining for Trx-1, MMP-2, MMP-9 and the NF- $\kappa$ B subunit p65 in the absence and presence of DATS $(25 \mathrm{mg} / \mathrm{kg}$ and $50 \mathrm{mg} / \mathrm{kg}$ ) in normal and mouse breast tumor tissues (up). Quantification of histochemical staining for Trx-1. Data were presented as means \pm SEM. ${ }^{*}, \mathrm{P}<0.05$; ${ }^{* *}, \mathrm{P}<0.01$, paired t-test (down). (B) The protein expression of trx-1 of tumor tissue by western blot assay. Three tumor mass were selected randomly in each group, then the homogenates were used for western blot assay.

\section{Discussion}

In the present study, we demonstrated that DATS was able to target Trx system and the decreased Trx-1 expression by DATS was responsible for the downregulation of a variety of metastasis-associated genes and suppression of breast cancer. In particular, the reduced form of Trx-1 was markedly lessened in the presence of DATS, indicating the cellular thiolbased redox state was influenced following DATS treatment. Among the components of thioredoxin system, the elevated level of TXNIP and diminished enzyme activity of TrxR mediated by DATS had additional effects on the direct target, Trx1 (Fig. 11). DATS has been elucidated to be a novel therapeutic agent for cancer treatment. Nevertheless, little or no evidence to our knowledge exists for the underlying mechanism linking anti-metastatic

\section{KARGER}


Fig. 9. DATS suppressed spontaneous metastasis of primary mammary fat pad tumors. (A) Immunohistochemical staining for Trx-1, MMP2, MMP-9 and the NF$\kappa \mathrm{B}$ subunit p65 in the absence and presence of DATS $(25 \mathrm{mg} / \mathrm{kg}$ and $50 \mathrm{mg} / \mathrm{kg}$ ) and $5 \mathrm{mg} / \mathrm{kg}$ cisplatin in normal and metastatic lung tissues (up). Quantification of histochemical staining for Trx-1, MMP-2, MMP9 and $N F-\kappa B$, Data were presented as means \pm SEM. * $\mathrm{P}<0.05$, paired t-test (down).

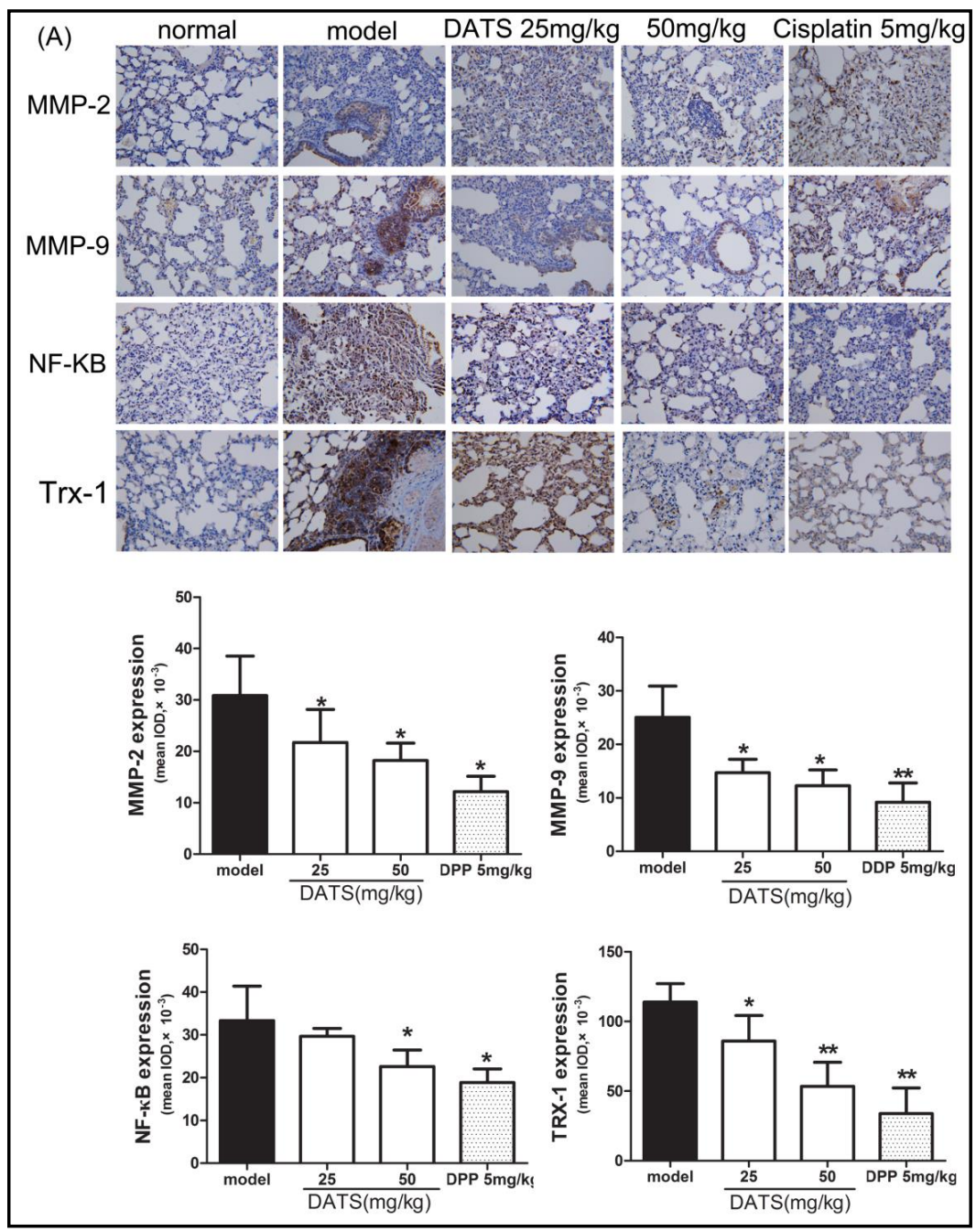

effect of DATS to its direct target. In the present study, we demonstrated that DATS was able to target Trx system and the decreased Trx- 1 expression by DATS was responsible for the downregulation of a variety of metastasis-associated genes and suppression of breast cancer metastasis. Therefore, targeting Trx-1 by DATS emerges as a novel strategy for the treatment of metastasis.

In contrast to normal cells, tumor cells are characterized by disrupted redox balance and deregulated redox signaling that are strongly implicated in malignant progression and resistance to treatment [36]. Tumor cells make use of a series of antioxidant systems to survive, proliferate, and metastasize, in which Trx system is thought to play a protective role against oxidative stress and inhibit redox stress-induced cell death in cancer [37]. The classic Trx system, composed of Trx, TrxR and NADPH, defines a major pathway of cellular thiolbased redox modulation [38]. Trx-1 is known to be a critical reducing enzyme that cleaves disulfides in proteins and acts as an S-denitrosylase [39]. Our results demonstrated that Trx1 protein expression in the breast cancer tissues was much higher than that in paracancerous tissues using a tissue microarray. Also, the elevated level of Trx-1 was closely associated with tumor invasion, lymph node metastasis as well as characteristics of TNBC, suggesting the expression of Trx-1 can recognized as an indicator of highly aggressive breast cancer. Breast cancer patients with lymph node metastasis displayed more nuclear expression of Trx-1 than non-metastasis patients. Thus, Trx-1 nuclear expression can be utilized as a diagnostic and prognostic marker for metastatic breast cancer, which was confirmed by the different Trx-1 expression patterns of high (MDA-MB-231 and Hs578T) and low metastatic (MCF-7 
Fig. 10. The effect of DATS on breast caner metastasis was mediated by Trx-1. (A) Protein expression of Trx-1, TXNIP and TrxR in MDA-MB-231 cell lysates following the transfection of control siRNAs or Trx-1 siRNAs in the absence or presence of $20 \mu \mathrm{M}$ of DATS. GAPDH was used as a loading control. (B) Representative images of horizontal migration of MDA-MB-231 cells following the transfection of control siRNAs or Trx-1 siRNAs in the absence or presence of $20 \mu \mathrm{M}$ of DATS. (C) Quantification of horizontal migration of MDAMB-231 cells following the transfection of control siRNAs or Trx-1 siRNAs in the absence or presence of $20 \mu \mathrm{M}$ of DATS. (D) Protein expression or phosphorylation of Erk, RhoA, Rac1/2/3, FAK, MMP-2 and MMP-9 in MDA-MB-231 cell lysates following the transfection of control siRNAs or Trx-1 siRNAs in the absence or presence of $20 \mu \mathrm{M}$ of DATS. GAPDH was used as a loading control.

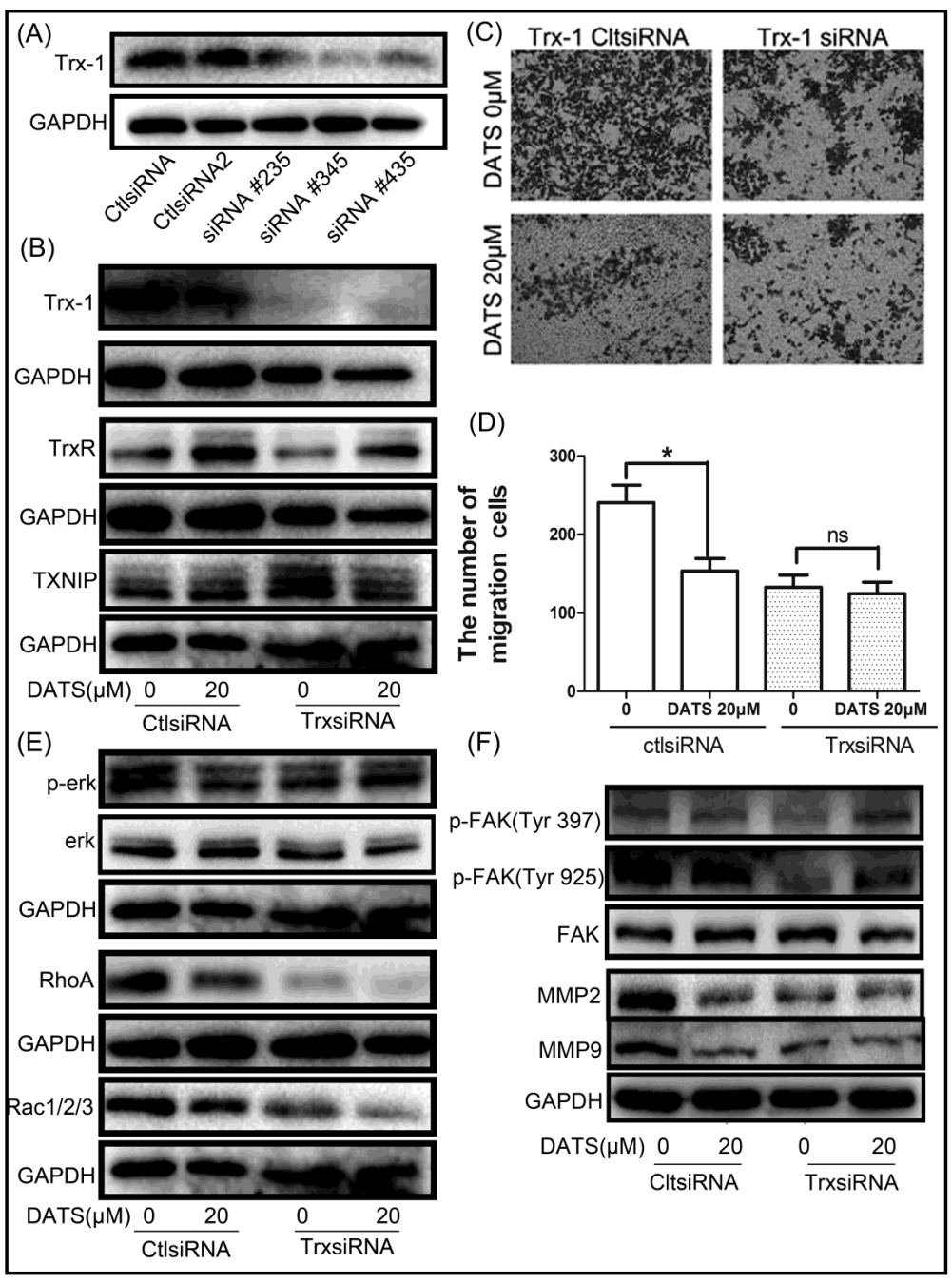

and ZR-75-1) cell lines. MDA-MB-231 and Hs578T cells (TNBC cell lines) with elevated Trx-1 expression provided efficient tools for the screening of drug candidate.

The anti-cancer effect of PX-12 clearly elucidated that inhibition of Trx-1 resulted in the decreased metastatic potential of MDA-MB-231 cells. Fluorescence staining of the actin cytoskeleton uncovered the PX-12 accelerated the conversion of fusiform and triradiate morphotypes to oval and round morphotypes, suggesting Trx-1 interacts with cytoskeleton proteins (e.g. RhoA and Rac1/2/3) that are related to cell motility. To this end, seeking the effective ingredient from flavouring and medicinal agents on the basis of the structure of PX-12 appeared to be a reliable route. DATS with a diallyl and three sulfur atoms required lowest binding energy to reduced Trx-1 based on computational docking results, suggesting the highest affinity exists between DATS and reduced Trx-1. The docking data were verified by western blot results, which illustrated that DATS attenuated Trx-1 expression in a dosedependent manner and diminished DTT-induced reduced Trx-1. Trx-1 in its reduced form plays a key role in redox modulation of many transcription factors (e.g. NF- $\kappa B$ and ASK1 ) and enables it to translocate into nucleus $[38,40]$. Thus, the elevated Trx-1 level in the nuclei provides a more-reducing environment required for DNA binding by transcription factors. DATS contributed to decreased nuclear translocation of Trx-1 and thus limited the interaction with downstream transcription factors that control the expression of a host of metastasis-associated genes.

TrxR is known as a redox-active enzyme equipped with a selenocysteine residue essential for catalysis [41]. In the process of redox reactions, thiols within the catalytic center of Trx-1 form a covalent disulfide bond that is reduced by TrxR [42]. Since TrxR is the only enzyme 


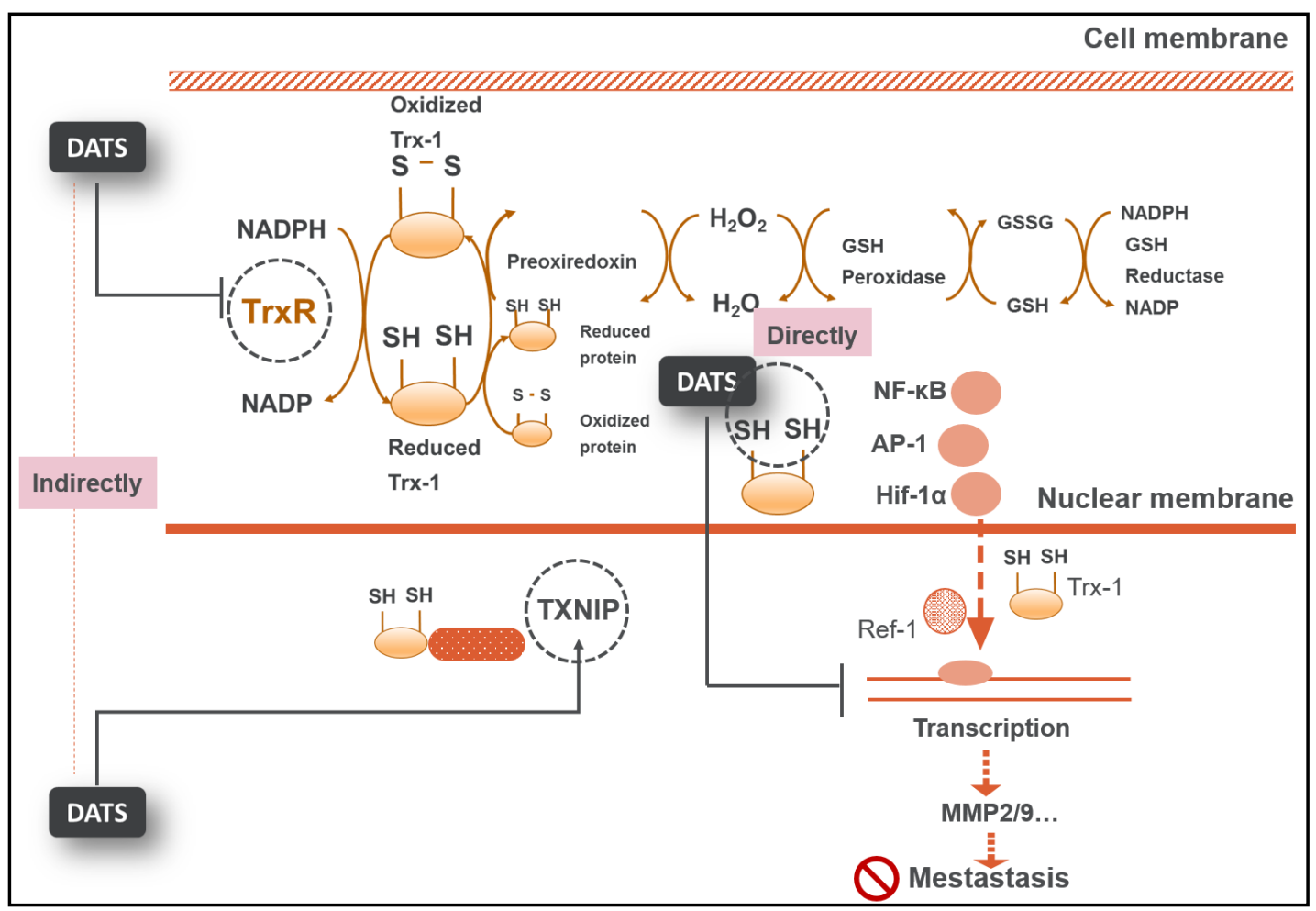

Fig. 11. A possible anti-metastasis mechanism of DATS in cancer cells. DATS exerted anti-metastatic effect on TNBC by targeting Trx system and attenuating reduced Trx-1 expression. The decreased Trx-1 expression by DATS was responsible for the downregulation of a variety of metastasis-associated proteins (MMP2, MMP9, etc.).

known to catalyze the reduction of Trx-1, it is reasonable that alterations in TrxR activity is likely to regulate some of the activities of Trx-1[43]. Importantly, high expression of TrxR is associated with a poor cancer prognosis in clinic $[16,44]$. Our data demonstrated that the enzyme activity of TrxR was dropped though no obvious change in expression was observed following the treatment of DATS. In addition, DATS resulted in augmented expression of TXNIP that is an endogenous inhibitor of Trx-1. Collectively, DATS is able to regulate TrxR and TXNIP, exerting additive effects with suppressing Trx-1 expression. Thus, Trx system in breast cancer can be targeted by DATS.

Experimental metastasis model of breast cancer assesses the ability of tumor cells to arrest, extravasate, and grow in prevalent organs such as lung and liver following intravenous injection [45]. The model was utilized in our study to explore the effects of DATS on late stage metastasis. Both the results of macrometastasis on the surface of lung and liver and micrometastasis by H\&E staining revealed that DATS was capable of inhibiting the production of experimental metastasis in nude mice. Immunohistochemical profiles of lung tissue revealed that DATS diminished the expression of Trx- 1 of metastatic breast cancer cells, which was consistent with what we observed in vitro. Additionally, NF- $\kappa \mathrm{B}$ as the key transcription factor that associated with Trx-1 was downregulated by DATS, which was accompanied by the reduction of metastasis-related genes MMP2 and MMP9. Clearly, targeting Trx- 1 by DATS leads to a synergistic effect with MMP2/9 in limiting the experimental metastasis of breast cancer. In consistent with experimental metastasis, DATS also resulted in a decrease in spontaneous metastasis model that mimics whole metastatic process. Although DAT-treated group shared similar average tumor size and weight with non-treated group, we did find that DATS contributed to decreased expression of Trx-1 in primary tumor and lung tissue. This suggests that the inhibitory effect of DATS on Trx-1 expression existed in the whole metastatic process, but Trx-1 inhibition-mediated apoptosis function did not occur in time.

We have previously shown that DATS emerges as a potential anti-metastatic agent via suppressing MMP2/9 in breast cancer cells. Here, we demonstrated that Trx-1 is likely to be 
the direct target of DATS with special disulfide bond structure. Furthermore, silence of Trx1 by siRNA could achieve similar effect with DATS such as blocked NF- $\kappa B$ activity, reduced the expression of metastasis-associated genes (MMP2/9, RhoA, Rac1/2/3) and decreased tumor cell migration. Nevertheless, such big difference between DATS treated group and non-treated group was abrogated in combination with Trx-1 siRNA, indicating the changes in the phenotypes of breast cancer cells by DATS was mediated by Trx-1. Consistently, in vivo, the supressed expression of MMP2/9 and NF- $\kappa$ B following the treatment of DATS coincided with the decreased level of Trx-1. In future, Trx-1 knockout mice can be utilized to further confirm DATS induced anti-metastasis effect in breast cancer and to verify the critical role of Trx-1 in breast cancer progression.

Taken together, our findings revealed the significant role of a potent organosulfur compound, DATS, in controlling TNBC metastasis. The data presented here demonstrated Trx-1 acts as the direct target of DATS, mediating the anti-metastasis effect of DATS via downregulating a series of metastasis-associated genes. Therefore, the efficacy of DATS in suppressing TNBC metastasis via targeting Trx system may provide new insight into therapeutic interventions for breast cancer metastasis.

\section{Abbreviations}

TNBC (triple-negative breast cancer); ER (estrogen receptor); PR (progesterone receptor); HER-2 (Human epidermal growth factor receptor 2); ROS (reactive oxygen species); Trx (Thioredoxin); TrxR (thioredoxin reductase); NADPH (nicotinamide adenine dinucleotide phosphate); TXNIP (thioredoxin-interacting protein); ASK1 (apoptosis signal-regulating kinase 1); HIF-1 $\alpha$ (hypoxia-inducible factor 1 $\alpha$ ); OSCc (Organic Sulphur compounds); DAS (diallyl sulphide); DADS (diallyl disulphide); DATS (diallyl trisulphide); DTT (DL-dithiothretiol); IAA (Iodoacetic acid); TMA (Tissue Microarray).

\section{Acknowledgements}

This project was supported in part by National Natural Science Foundation of China (81403260, 81573859, 81673725 and 81673648), China Postdoctoral Science Foundation (2014M551639 and 2016M601865), Natural Science Foundation of Jiangsu Province (BK20171097), Science and technology project of Jiangsu Provincial Bureau of traditional Chinese Medicine (YB2017033), Natural Science Foundation of Higher School of Jiangsu Province (17KJA360003), Postdoctoral funding in Jiangsu Province (1401138C), 2013' Program for Excellent Scientific and Technological Innovation Team of Jiangsu Higher Education, Top-notch Academic Programs Project of Jiangsu Higher Education Institutions (PPZY2015A070), and a project of the Priority Academic Program Development of Jiangsu Higher Education Institutions (PAPD). YL supervised the study; YPL, YZ and ZHW conceived and designed experiments; YPL, PTZ, XBS and SLW performed experiments; YPL, JSR, YZC and YLS analyzed data; YPL, YZ and YL wrote the manuscript.

\section{Disclosure Statement}

No conflict of interests exists.

\section{References}

1 Galeone C, Pelucchi C, Dal Maso L, Negri E, Montella M, Zucchetto A, Talamini R, La Vecchia C: Allium vegetables intake and endometrial cancer risk. Public Health Nutr 2009;12:1576-1579.

-2 Liu Y, Zhu P, Wang Y, Wei Z, Tao L, Zhu Z, Sheng X, Wang S, Ruan J, Liu Z, Cao Y, Shan Y, Sun L, Wang A, Chen W, Lu Y: Antimetastatic Therapies of the Polysulfide Diallyl Trisulfide against Triple-Negative Breast Cancer (TNBC) via Suppressing MMP2/9 by Blocking NF-kappaB and ERK/MAPK Signaling Pathways. PLoS One 2015;10:e0123781. 


\section{Cellular Physiology Cell Physiol Biochem 2018;50:1945-1963 \begin{tabular}{l|l|l|l|l}
\hline DOI: 10.1159/000494874 2018 The Author(s). Published by S. Karger AG, Basel & and Biochemistry
\end{tabular}

Liu et al.: Targeting Trx-1 Limits Metastasis

- Puccinelli MT, Stan SD: Dietary Bioactive Diallyl Trisulfide in Cancer Prevention and Treatment. Int J Mol Sci 2017;18.

4 Li Y, Zhang J, Zhang L, Si M, Yin H, Li J: Diallyl trisulfide inhibits proliferation, invasion and angiogenesis of osteosarcoma cells by switching on suppressor microRNAs and inactivating of Notch-1 signaling. Carcinogenesis 2013;34:1601-1610.

5 Torre LA, Bray F, Siegel RL, Ferlay J, Lortet-Tieulent J, Jemal A: Global cancer statistics, 2012.CA Cancer J Clin 2015;65:87-108.

6 Weigelt B, Peterse JL, van 't Veer LJ: Breast cancer metastasis: markers and models. Nat Rev Cancer 2005;5:591-602.

7 Yates LR, Knappskog S, Wedge D, Farmery JHR, Gonzalez S, Martincorena I, Alexandrov LB, Van Loo P, Haugland HK, Lilleng PK, Gundem G, Gerstung M, Pappaemmanuil E, Gazinska P, Bhosle SG, Jones D, Raine K, Mudie L, Latimer C, Sawyer E, Desmedt C, Sotiriou C, Stratton MR, Sieuwerts AM, Lynch AG, Martens JW, Richardson AL, Tutt A, Lonning PE, Campbell PJ: Genomic Evolution of Breast Cancer Metastasis and Relapse. Cancer Cell 2017;32:169-184 e167.

$>8$ Palma G, Frasci G, Chirico A, Esposito E, Siani C, Saturnino C, Arra C, Ciliberto G, Giordano A, D’Aiuto M: Triple negative breast cancer: looking for the missing link between biology and treatments. Oncotarget 2015;6:26560-26574.

-9 Lehmann BD, Bauer JA, Chen X, Sanders ME, Chakravarthy AB, Shyr Y, Pietenpol JA: Identification of human triple-negative breast cancer subtypes and preclinical models for selection of targeted therapies. J Clin Invest 2011;121:2750-2767.

10 Bianchini G, Balko JM, Mayer IA, Sanders ME, Gianni L: Triple-negative breast cancer: challenges and opportunities of a heterogeneous disease. Nat Rev Clin Oncol 2016;13:674-690.

11 Gucalp A, Traina TA: Triple-negative breast cancer: adjuvant therapeutic options. Chemother Res Pract 2011;2011:696208.

12 Holohan C, Van Schaeybroeck S, Longley DB, Johnston PG: Cancer drug resistance: an evolving paradigm. Nat Rev Cancer 2013;13:714-726.

13 Qu Y, Wang J, Ray PS, Guo H, Huang J, Shin-Sim M, Bukoye BA, Liu B, Lee AV, Lin X, Huang P, Martens JW, Giuliano AE, Zhang N, Cheng NH, Cui X: Thioredoxin-like 2 regulates human cancer cell growth and metastasis via redox homeostasis and NF-kappaB signaling. J Clin Invest 2011;121:212-225.

-14 Piskounova E, Agathocleous M, Murphy MM, Hu Z, Huddlestun SE, Zhao Z, Leitch AM, Johnson TM, DeBerardinis RJ, Morrison SJ: Oxidative stress inhibits distant metastasis by human melanoma cells. Nature 2015;527:186-191.

15 Leone A, Roca MS, Ciardiello C, Costantini S, Budillon A: Oxidative Stress Gene Expression Profile Correlates with Cancer Patient Poor Prognosis: Identification of Crucial Pathways Might Select Novel Therapeutic Approaches. Oxid Med Cell Longev 2017;2017:2597581.

16 Zhang J, Li X, Han X, Liu R, Fang J: Targeting the Thioredoxin System for Cancer Therapy. Trends Pharmacol Sci 2017;38:794-808.

17 He L, He T, Farrar S, Ji L, Liu T, Ma X: Antioxidants Maintain Cellular Redox Homeostasis by Elimination of Reactive Oxygen Species. Cell Physiol Biochem 2017;44:532-553.

18 Schwertassek U, Haque A, Krishnan N, Greiner R, Weingarten L, Dick TP, Tonks NK: Reactivation of oxidized PTP1B and PTEN by thioredoxin 1. FEBS J 2014;281:3545-3558.

19 Sun Y, Rowehl LM, Huang L, Mackenzie GG, Vrankova K, Komninou D, Rigas B: Phospho-ibuprofen (MDC917) suppresses breast cancer growth: an effect controlled by the thioredoxin system. Breast Cancer Res 2012;14:R20.

20 Sengupta R, Holmgren A: Thioredoxin and thioredoxin reductase in relation to reversible S-nitrosylation. Antioxid Redox Signal 2013;18:259-269.

-21 Holmgren A: Thioredoxin structure and mechanism: conformational changes on oxidation of the active-site sulfhydryls to a disulfide. Structure 1995;3:239-243.

22 Samaranayake GJ, Troccoli CI, Huynh M, Lyles RDZ, Kage K, Win A, Lakshmanan V, Kwon D, Ban Y, Chen SX, Zarco ER, Jorda M, Burnstein KL, Rai P: Thioredoxin-1 protects against androgen receptor-induced redox vulnerability in castration-resistant prostate cancer. Nat Commun 2017;8:1204.

-23 Tobiume K, Matsuzawa A, Takahashi T, Nishitoh H, Morita K, Takeda K, Minowa O, Miyazono K, Noda T, Ichijo H: ASK1 is required for sustained activations of JNK/p38 MAP kinases and apoptosis. EMBO Rep 2001;2:222-228.

24 Welsh SJ, Bellamy WT, Briehl MM, Powis G: The redox protein thioredoxin-1 (Trx-1) increases hypoxiainducible factor 1alpha protein expression: Trx-1 overexpression results in increased vascular endothelial growth factor production and enhanced tumor angiogenesis. Cancer Res 2002;62:5089-5095.

-25 Streicher KL, Sylte MJ, Johnson SE, Sordillo LM: Thioredoxin reductase regulates angiogenesis by increasing endothelial cell-derived vascular endothelial growth factor. Nutr Cancer 2004;50:221-231. 


\section{Cellular Physiology Cell Physiol Biochem 2018;50:1945-1963 and Biochemistry DOl: 10.1159/000494874 20 Publisher 2018 The Author(s). Published by S. Karger AG, Basel

Liu et al.: Targeting Trx-1 Limits Metastasis

26 Farina AR, Cappabianca L, DeSantis G, Di Ianni N, Ruggeri P, Ragone M, Merolle S, Tonissen KF, Gulino A, Mackay AR: Thioredoxin stimulates MMP-9 expression, de-regulates the MMP-9/TIMP-1 equilibrium and promotes MMP-9 dependent invasion in human MDA-MB-231 breast cancer cells. FEBS Lett 2011;585:3328-3336.

27 Kononen J, Bubendorf L, Kallioniemi A, Barlund M, Schraml P, Leighton S, Torhorst J, Mihatsch MJ, Sauter G, Kallioniemi OP: Tissue microarrays for high-throughput molecular profiling of tumor specimens. Nat Med 1998;4:844-847.

28 Kim J, Yu J: Interrogating genomic and epigenomic data to understand prostate cancer. Biochim Biophys Acta 2012;1825:186-196.

29 Chen J, Miller EM, Gallo KA: MLK3 is critical for breast cancer cell migration and promotes a malignant phenotype in mammary epithelial cells. Oncogene 2010;29:4399-4411.

-30 Sun Y, Rigas B: The thioredoxin system mediates redox-induced cell death in human colon cancer cells: implications for the mechanism of action of anticancer agents. Cancer Res 2008;68:8269-8277.

31 Zhang L, Duan D, Liu Y, Ge C, Cui X, Sun J, Fang J: Highly selective off-on fluorescent probe for imaging thioredoxin reductase in living cells. J Am Chem Soc 2014;136:226-233.

32 Zhang J, Yao J, Peng S, Li X, Fang J: Securinine disturbs redox homeostasis and elicits oxidative stressmediated apoptosis via targeting thioredoxin reductase. Biochim Biophys Acta 2017;1863:129-138.

33 Iorns E, Drews-Elger K, Ward TM, Dean S, Clarke J, Berry D, El Ashry D, Lippman M: A new mouse model for the study of human breast cancer metastasis. PLoS One 2012;7:e47995.

34 Feng X, Wu Z, Wu Y, Hankey W, Prior TW, Li L, Ganju RK, Shen R, Zou X: Cdc25A regulates matrix metalloprotease 1 through Foxo1 and mediates metastasis of breast cancer cells. Mol Cell Biol 2011;31:3457-3471.

-35 Baker AF, Dragovich T, Tate WR, Ramanathan RK, Roe D, Hsu CH, Kirkpatrick DL, Powis G: The antitumor thioredoxin-1 inhibitor PX-12 (1-methylpropyl 2-imidazolyl disulfide) decreases thioredoxin-1 and VEGF levels in cancer patient plasma. J Lab Clin Med 2006;147:83-90.

-36 Abdelsaid MA, Matragoon S, Ergul A, El-Remessy AB: Deletion of thioredoxin interacting protein (TXNIP) augments hyperoxia-induced vaso-obliteration in a mouse model of oxygen induced-retinopathy. PLoS One 2014;9:e110388.

-37 Raninga PV, Trapani GD, Tonissen KF: Cross Talk between Two Antioxidant Systems, Thioredoxin and DJ-1: Consequences for Cancer. Oncoscience 2014;1:95-110.

-38 Du Y, Zhang H, Zhang X, Lu J, Holmgren A: Thioredoxin 1 is inactivated due to oxidation induced by peroxiredoxin under oxidative stress and reactivated by the glutaredoxin system. J Biol Chem 2013;288:32241-32247.

-39 Anand P, Stamler JS: Enzymatic mechanisms regulating protein S-nitrosylation: implications in health and disease. J Mol Med (Berl) 2012;90:233-244.

40 Go YM, Jones DP: Redox control systems in the nucleus: mechanisms and functions. Antioxid Redox Signal 2010;13:489-509.

41 Zhong L, Arner ES, Holmgren A: Structure and mechanism of mammalian thioredoxin reductase: the active site is a redox-active selenolthiol/selenenylsulfide formed from the conserved cysteine-selenocysteine sequence. Proc Natl Acad Sci U S A 2000;97:5854-5859.

42 Mougiakakos D, Johansson CC, Jitschin R, Bottcher M, Kiessling R: Increased thioredoxin-1 production in human naturally occurring regulatory $\mathrm{T}$ cells confers enhanced tolerance to oxidative stress. Blood 2011;117:857-861.

43 Mustacich D, Powis G: Thioredoxin reductase. Biochem J 2000;346 Pt 1:1-8.

44 Bhatia M, McGrath KL, Di Trapani G, Charoentong P, Shah F, King MM, Clarke FM, Tonissen KF: The thioredoxin system in breast cancer cell invasion and migration. Redox Biol 2016;8:68-78.

45 Mohanty S, Xu L: Experimental metastasis assay. J Vis Exp 2010;pii: 1942. 